\title{
The Kondo Lattice Models and Magnetism for Cerium and Uranium Systems
}

\begin{abstract}
B. COQBlin*
Laboratoire de Physique des Solides, UMR 8502, CNRS, Université Paris-Sud, 91405-Orsay, France

The interplay between the Kondo effect and magnetism is important in anomalous rare-earth and actinide systems and a brief review of the different Kondo lattice models is presented here. The first studied case is the classical Kondo lattice model with $S=1 / 2$ spins for the $4 f$ localized electrons, which describes the strong competition between the Kondo effect and magnetic ordering leading to small ordering temperatures less than roughly $10 \mathrm{~K}$. The second reviewed case describes the underscreened Kondo lattice model with $S=1$ spins for the $5 f$ localized electrons, which can account for the coexistence of both the Kondo effect and the ferromagnetic order and, therefore, the large Curie temperatures observed in actinide compounds like UTe and $\mathrm{NpNiSi}_{2}$; the question of the delocalization of the $5 f$ electrons is also discussed here. The last case describes the competition between the "spin glass" behaviour, the Kondo effect and the magnetic ordering in disordered cerium alloys like $\mathrm{CeNi}_{x} \mathrm{Cu}_{1-x}$ or $\mathrm{CeRh}_{x} \mathrm{Pd}_{1-x}$ and several models used for the spin glass state are reviewed, as well as the occurrence of magnetic glass clusters.
\end{abstract}

PACS numbers: 75.30.Mb, 75.20.Hr, 05.50.+q, 64.60.Cn

\section{Introduction to Kondo lattice models}

The competition between the Kondo effect and magnetism plays a very important role in anomalous rare-earth and actinide systems and a brief review of our recent works on three interesting features is presently here, namely the $S=1 / 2$ Kondo lattice model, the underscreened Kondo lattice model and finally the spin glassKondo competition.

The first extensively studied case is the classical Kondo lattice problem, in which the spins $S=1 / 2$ of the localized $4 f$ electrons (or holes) corresponding to the $4 f^{1}$ (or $4 f^{13}$ ) configuration are completely screened at very low temperatures by the spins $s=1 / 2$ of the conduction electrons, leading to a strong Kondo-magnetism competition, which has been firstly described by the Doniach diagram [1] and extensively reviewed for instance in Refs. [2-7]. It is also well recognized that the Kondo compounds can be heavy fermion systems which do not order magnetically at very low temperatures, like $\mathrm{CeAl}_{3}$, or that they can order magnetically with a small ordering temperature generally smaller than $10 \mathrm{~K}$, like $\mathrm{CeAl}_{2}$. Moreover, the transition from magnetic order to a non-magnetic heavy fermion state has been experimentally observed with increasing pressure in many cerium compounds, such as $\mathrm{CeAl}_{2}$ [8] or $\mathrm{CeRh}_{2} \mathrm{Si}_{2}$ [9]. A similar effect has been observed in $\mathrm{YbCu}_{2} \mathrm{Si}_{2}$ [10] or in other ytterbium compounds, where the Néel temperature starts from zero at a given pressure and increases rapidly with pressure, since pressure has an opposite effect in ytterbium compounds than in cerium compounds.

The normal Kondo lattice model for $S=1 / 2$ spins with both an intrasite Kondo exchange interaction and an

\footnotetext{
* corresponding author; e-mail: coqblin@lps.u-psud.fr
}

intersite magnetic exchange interaction between neighbouring localized spins has been extensively studied within the mean-field approximation [11-14].

The resulting Hamiltonian of the system is given by

$$
\begin{aligned}
H & =\sum_{\boldsymbol{k} \sigma} \varepsilon_{\boldsymbol{k}} n_{\boldsymbol{k} \sigma}^{\mathrm{c}}+\sum_{i \sigma} E_{0} n_{i \sigma}^{f}+J_{\mathrm{K}} \sum_{i} \boldsymbol{s}_{i} \cdot \boldsymbol{S}_{i} \\
& +J_{H} \sum_{\langle i j\rangle} \boldsymbol{S}_{i} \cdot \boldsymbol{S}_{j}
\end{aligned}
$$

where $\varepsilon_{\boldsymbol{k}}$ is the energy of the conduction band, $J_{\mathrm{K}}>0$ is the Kondo coupling between the localized spin $\boldsymbol{S}_{i}$ and the spin $\boldsymbol{s}_{i}$ of a conduction electron at the same site and $J_{H}$ is the interaction between nearest-neighboring localized spins. We consider here the case of choosing $J_{H}$ to be positive or negative for antiferromagnetic or ferromagnetic intersite interactions. We consider firstly here the case of choosing $J_{H}$ to be positive, implying that intersite interactions are antiferromagnetic, as it is the case of most cerium compounds [13-15]. We have studied the Kondo effect and the short-range magnetic correlations by considering respectively the two operators $\lambda=\left\langle c_{i \sigma}^{*} f_{i \sigma}\right\rangle$ and $\Gamma=\left\langle f_{i \sigma}^{*} f_{j \sigma}\right\rangle$. We have computed the Kondo temperature $T_{\mathrm{K}}$ and the correlation temperature $T_{\text {cor }}$ at which the short-range magnetic correlations disappear and we have shown that the Kondo effect occurs for a large $J_{\mathrm{K}}$ value and that is destroyed by an increase of $J_{H}$. The Kondo effect tends also to be destroyed by a decrease of the number of conduction electrons, called the "exhaustion" effect $[13,14,16]$.

Then, in order to describe the competition between the Kondo effect and the antiferromagnetic order, we have used again the same mean-field approximation by taking two order parameters $\lambda_{i \sigma}$ for the Kondo effect and then the average magnetization for each sublattice of the antiferromagnetic lattice [15]: this model gives a transi- 
tion versus $J_{\mathrm{K}}$ from an antiferromagnetic ordering to a Kondo phase, which can account roughly for the effect of pressure in the Doniach diagram. Another conclusion is that the Kondo temperature for the lattice can be different and generally much smaller than the single-impurity one. The comparison with experiment is not easy, but it was suggested that such effets can be observed in cerium systems like $\mathrm{CeRh}_{2} \mathrm{Si}_{2}$ compound [9, 17, 18].

In conclusion of this section, the Kondo lattice model explains well the behavior of many cerium, ytterbium or other anomalous rare-earth systems. Thus, the effect of pressure is often to change a magnetically ordered cerium compound to a non-magnetic compound with a large heavy-fermion character and an opposite effect has been observed in some ytterbium compounds, in good agreement with theory. A similar variation of the magnetism of $\mathrm{Ce}$ or $\mathrm{Yb}$ in alloys has been also observed when there is a change in the concentration of the matrix.

Recently a new and very interesting effect has been also obtained by tuning the dimensionality of the heavy fermion compound $\mathrm{CeIn}_{3}$ [19]. In fact, artificial superlattices of the antiferromagnetic heavy fermion compound $\mathrm{CeIn}_{3}$ and of the conventional non magnetic metal $\mathrm{LaIn}_{3}$ have been experimentally studied. The heavy fermion compound $\mathrm{CeIn}_{3}$ exhibits an antiferromagnetic ordering at the Néel temperature $T_{\mathrm{N}}=10 \mathrm{~K}$, which is destroyed by applying pressure. By reducing the thickness of the $\mathrm{CeIn}_{3}$ layers, the Néel temperature decreases and finally the magnetic order was suppressed; similarly, the Fermi liquid coefficient of the low temperature resistivity increases. This very interesting result is finally interpreted by saying that the decrease of the dimensionality from 3D to $2 \mathrm{D}$ has the same effect as the increase of pressure, giving, therefore, a decrease and finally the disappearance of the magnetic ordering [19]. This effect is very fascinating and can be reproduced in other heavy fermion compounds.

\section{The underscreened Kondo lattice model applied to uranium compounds}

We have seen in the previous section that there exists a strong Kondo-magnetic order competition in cerium or ytterbium compounds. The situation is different in actinide systems, where the $5 f$ electrons are clearly in a crossover region between localized and itinerant behavior and are less localized than the $4 f$ electrons of rare earths. Thus, several magnetic behaviors have been observed in actinide systems.

In the series of actinide metals, a clear magnetism occurs only for curium in the middle of the series, while a spin fluctuation model has been developed to account for the transport properties of $\mathrm{Np}$ and $\mathrm{Pu}$ metals [20]. Similarly, some actinide compounds are clearly magnetic, while other ones like $\mathrm{UAl}_{2}$ or $\mathrm{PuAl}_{2}$ have been well described by the spin fluctuation model [21]. Uranium compounds are often in an intermediate situation for the localization of the $5 f$ electrons and uranium monochalcogenides provide a good example of the complexity of the
$5 f$ electrons, which appear to be itinerant in US, localized in UTe and in between in USe [22, 23]. A model involving this "dual nature" with two localized $5 f$ electrons and one itinerant has been introduced to describe some uranium compounds [24].

Uranium compounds, UTe [23], $\mathrm{UCu}_{0.9} \mathrm{Sb}_{2}$ [25] or $\mathrm{UCo}_{0.5} \mathrm{Sb}_{2}[26]$ present a ferromagnetic ordering at very large Curie temperatures (equal respectively to $T_{\mathrm{c}}=$ $102 \mathrm{~K}, 113 \mathrm{~K}$ and $64.5 \mathrm{~K}$ ) and show also a $\log T$ Kondo-type decrease of the resistivity above $T_{\mathrm{c}}$. A similar behavior has been recently observed in neptunium compounds $\mathrm{NpNiSi}_{2}$ [27] and $\mathrm{Np}_{2} \mathrm{PdGa}_{3}$ [28].

We have recently developed an underscreened Kondo lattice (UKL) model $[29,30]$ to account for the coexistence between the Kondo effect and the ferromagnetic ordering observed in some uranium compounds. In fact, we present here firstly the UKL model [29] and then a recent improved description to better account for a weak delocalization of the $5 f$ electrons $[30,31]$.

It is difficult to interpret different experiments in UTe, but clearly the magnetic moments deduced from magnetic susceptibility experiments in this compound are relatively close to the free ion values of uranium, which implies that the $5 f$ electrons are well localized in UTe [23] and can be correctly described within a $5 f^{2}$ configuration.

In the UKL model [29], the two $5 f$-electrons are bound into a spin $S_{f}=1$. The Hamiltonian is similar to that of Eq. (1), with the following essential difference: the localized spins are here $\boldsymbol{S}=1$ and the second term of the Hamiltonian (1) can be written now as $\sum_{i \sigma \alpha} E_{0} n_{i \sigma}^{f_{\alpha}}$, in order to describe the energy $E_{0}$ of the two $5 f$ localized electrons (defined by the notation $\alpha=1,2$ ) of the $5 f^{2}$ configuration of uranium atoms. Then, the intersite interaction is ferromagnetic with $J_{H}<0$.

In the UKL model of Ref. [29], we have used the mean field approximation by introducing the Kondo operators

$$
\widehat{\lambda}_{i \sigma}=\sum_{\alpha} \widehat{\lambda}_{i \sigma}^{\alpha}=\sum_{\alpha}\left\langle c_{i \sigma}^{+} f_{i \sigma}^{\alpha}\right\rangle,
$$

which couples conduction and $f$ electrons at the same site.

Moreover, in order to describe the magnetic properties of the system, we introduce also the magnetization operators for both $f$ - and $c$-electrons:

$$
\begin{aligned}
& M_{i}=S_{i}^{z}=\frac{1}{2}\left(n_{i \uparrow}^{f}-n_{i \downarrow}^{f}\right), \\
& m_{i}=\sigma_{i}^{z}=\frac{1}{2}\left(n_{i \uparrow}^{\mathrm{c}}-n_{i \downarrow}^{\mathrm{c}}\right) .
\end{aligned}
$$

The non-zero values of $\langle M\rangle$ and $\langle m\rangle$ describe the magnetic phase, while a non-zero $\lambda_{\sigma}$ describes the Kondo effect and the formation of the heavy-fermion state.

The detailed calculations can be found in Ref. [29]. The Kondo temperature $T_{\mathrm{K}}$ becomes finite only above a critical value $J_{\mathrm{K}}^{\mathrm{c}}$ and then increases rapidly for larger values of $J_{\mathrm{K}}$. On the other hand, the Curie temperature, $T_{\mathrm{c}}$, is finite for all studied values of $J_{\mathrm{K}}$. The two curves of $T_{\mathrm{K}}$ and $T_{\mathrm{c}}$ cross slightly above $J_{\mathrm{K}}^{\mathrm{c}}$ and, for larger values of $J_{\mathrm{K}}, T_{\mathrm{K}}$ is always larger than $T_{\mathrm{c}}$. 
The resulting "ferromagnetic-Kondo" diagram, which is given in Refs. [18, 29], is completely different from the Doniach diagram derived for the Kondo lattice model appropriate for cerium or ytterbium compounds and represents really a new result for the study of magnetic actinide compounds.

The ferromagnetic uranium monochalcogenides UTe, USe and US have been studied at very high pressures $[32,33]$ : the Curie temperature of UTe increases with pressure up to a maximum at roughly $7 \mathrm{GPa}$ and then decreases with larger pressures $[32,33]$. On the opposite, the Curie temperature of US is continuously decreasing with pressure and $T_{\mathrm{c}}$ of USe remains constant up to $10 \mathrm{GPa}$ and decreases rapidly at higher pressures [32].

The initial increase of $T_{\mathrm{c}}$ with pressure is clearly explained by our model, since $J_{\mathrm{K}}$ increases with increasing pressure. On the other hand, the maximum and the decrease of $T_{\mathrm{c}}$ at higher pressure cannot be understood in the UKL model and this decrease is connected to a weak delocalization of the $5 f$ electrons. Similarly, the decrease of $T_{\mathrm{c}}$ in US compound corresponds to a continuous delocalisation of the $5 f$ electrons which are already itinerant at normal pressure. The case of USe is intermediate, with $5 f$ electrons between localized and itinerant at normal pressure. Sheng and Cooper [22] have performed $a b$ initio band calculations which can account for the pressure dependence of $T_{\mathrm{c}}$ in uranium monochalcogenides.

Recently, in order to study a possible weak delocalization, we have studied the more general underscreened Anderson lattice (UAL) model, which starts from two $f$-electrons, with operators $f_{1 \sigma}$ and $f_{2 \sigma}$. Then we have used the Schrieffer-Wolff transformation and we have obtained a new Hamiltonian which contains both the classical $S=1$ exchange Kondo-type term and also a new $f$-band term with a finite $f$-band width proportional to $J_{\mathrm{K}}[31]$. The behavior of $T_{\mathrm{c}}$ and $T_{\mathrm{K}}$ presents two important differences with respect to the previous plots obtained with the UKL model [29]: first, $T_{\mathrm{c}}$ can pass through a maximum above roughly $J_{\mathrm{K}}^{\mathrm{c}}$ and decreases after with increasing $J_{\mathrm{K}}$; second, in a certain $J_{\mathrm{K}}$ range, two values of $T_{\mathrm{K}}$ are obtained and $\lambda$ is non-zero between these two temperatures. The first effect can account for the maximum of $T_{\mathrm{c}}$ observed in UTe at high pressures. The second effect is connected to the fact that the Kondo screening of $S=1$ spins cannot be completed at very low temperatures [34]; at present there is no experimental evidence in favor or in contrast of such an effect in actinide compounds at very low temperatures.

The previous calculations have been performed by assuming a fixed total number $n_{f}=2$ of $5 f$ electrons. But in fact, in order to study the delocalization of the $5 f$ electrons from a localized to an itinerant behavior, it is necessary to decrease the total number of $5 f$ electrons. A more complete mean-field treatment describing both the Kondo effect and the magnetism can be performed within the underscreened Anderson lattice Hamiltonian, in order to have a better description of the transition from the localized to the itinerant behavior of the $5 f$ electrons in actinide systems.

\section{The spin glass-Kondo-magnetic order competition}

The competition between the Kondo effect, the spin glass and a magnetic order has been observed in disordered cerium or uranium alloys. The first studied case is the $\mathrm{CeNi}_{x} \mathrm{Cu}_{1-x}$ alloys [35-39], but recently the $\mathrm{CeRh}_{x} \mathrm{Pd}_{1-x}$ alloys $[40,41]$ have been also studied and a new cluster glass state has been identified recently in these two cases. In fact, a careful experimental study of $\mathrm{CeRh}_{x} \mathrm{Pd}_{1-x}$ alloys shows the presence of the Kondo clusters near $x=0.8$ when the ferromagnetic phase is very close to disappear [40].

A spin glass-Kondo competition has been also observed in $\mathrm{Ce}_{2} \mathrm{Au}_{1-x} \mathrm{Co}_{x} \mathrm{Si}_{3}$ alloys [42] and in some disordered uranium alloys such as $\mathrm{UCu}_{5-x} \mathrm{Pd}_{x}$ [43] or $\mathrm{U}_{1-x} \mathrm{La}_{x} \mathrm{Pd}_{2} \mathrm{Al}_{3}[44]$.

The well known $\mathrm{CeNi}_{x} \mathrm{Cu}_{1-x}$ alloys are at low temperatures antiferromagnetic for low $\mathrm{Ni}$ concentration ( $x$ small) and have a Kondo behavior for low $\mathrm{Cu}$ concentration ( $x$ large). But the experimental situation is more complicate in the intermediate concentration range, typically for $x$ between 0.4 and 0.7 , where it was firstly shown that, when temperature decreases, there are successively a spin-glass phase and then a ferromagnetic one $[35,36]$.

But, very recently, these alloys have been investigated by $\mu \mathrm{SR}$ spectroscopy or small angle neutron scattering (SANS) or magnetic experiments leading to the determination of hysteresis loops [37-39]. The experimental results have been analyzed in terms of "cluster glass" (CG). Moreover, there is no clear indication of a Curie temperature $T_{\mathrm{c}}$ from ac-susceptibility and specific heat measurements. Thus, the proposed phase diagram in the intermediate concentration range consists firstly in the presence of an intermediate magnetic state where dynamic clusters develop with decreasing temperature below the paramagnetic state; then, the volume fraction of these clusters increases as temperature decreases and they become frozen at very low temperatures. Thus, recent experiments have shown the occurrence of an inhomogeneous "cluster spin glass", followed, with decreasing temperature, by an inhomogeneous ferromagnetic (IFM) order, with a percolative evolution of the clusters from the CG state to the IFM state $[38,39]$.

On the other hand, there has been an extensive effort to understand the complicate competition between the Kondo effect, the spin glass and magnetic orderings in such disordered cerium alloys. The calculation starts from a Hamiltonian similar to that given by Eq. (1), including the same intra-site Kondo interaction term with an exchange integral $J_{\mathrm{K}}$ and a different last term corresponding to the spin glass phase given by

$$
H_{\mathrm{SG}}=\sum_{i, j} J_{i j} S_{f i}^{z} S_{f j}^{z} .
$$

There are different calculations for the spin glass- 
Kondo interaction, but here we will present a brief review of our different calculations on this subject. The main difference between our recent works lies in the approach used to describe the spin glass and also in the comparison between our models and experimental results.

In our first calculation, we have taken the SherringtonKirkpatrick model [45] for the exchange intersite integrals which are assumed to be randomly distributed with a Gaussian distribution. The first model assumes a zero mean value of the Gaussian distribution in order to study only the Kondo-spin glass interaction and we have, therefore, obtained a phase diagram showing first a spin glass phase and then a Kondo phase with increasing $J_{\mathrm{K}}[46]$. Then, in order to obtain a more complex phase diagram with a ferromagnetic [47] or an antiferromagnetic [48] phase occurring at low temperatures for small $J_{\mathrm{K}}$ values, we have taken the same model but with a non-zero mean value $J_{0}$ of the Gaussian distribution. Thus, it results that the Kondo phase obtained for large $J_{\mathrm{K}}$ values is still there, but that there is a competition between the spin glass phase and the magnetic phase for smaller $J_{\mathrm{K}}$ values. However, in the ferromagnetic case, when $J_{\mathrm{K}}$ is typically of order $J_{0}$, we have obtained with decreasing temperature successively a ferromagnetic phase, then a mixed ferromagnetic-spin-glass phase and finally a spin glass phase. The evidence of a spin-glass phase at very low temperatures is in disagreement with experimental results previously described for $\mathrm{CeNi}_{x} \mathrm{Cu}_{1-x}$ alloys.

Thus, we have firstly considered another spin glass approach which introduces a generalization of the Mattis model [49] and which represents an interpolation between ferromagnetism and a highly disordered spin glass. The detailed calculations can be found in Ref. [50] and here we start from exchange integrals between spins on different sites $i$ and $j$ given by

$$
J_{i j}=\frac{1}{N} \sum_{\mu} J \xi_{i}^{\mu} \xi_{j}^{\mu}
$$

where the $\xi_{i}^{\mu}= \pm 1(\mu=1,2, \ldots, p ; i=1,2, \ldots, N)$ are independent randomly distributed variables, with an equal probability for $\xi_{i}^{\mu}$ to be equal to +1 or -1 . The critical parameter is here the ratio $a=p / N$, which gives an estimation of the relative importance of the ferromagnetic and spin glass phases for small $J_{\mathrm{K}}$ values; for large $J_{\mathrm{K}}$ values, the Kondo phase is always present.

For a small value of $a$, typically $a=0.04$, we have obtained, with decreasing temperature, a spin-glass phase, then a mixed ferromagnetic-spin-glass phase and finally a ferromagnetic phase with decreasing temperature for a relatively small $J_{\mathrm{K}} / J$ ratio. This theoretical result accounts better for the experimental phase diagram of $\mathrm{CeNi}_{x} \mathrm{Cu}_{1-x}$ alloys, with in particular the ferromagnetic phase below the spin-glass phase at the lowest temperatures, as shown on the phase diagram given in Ref. [50].

Then, we have developed more recently the van Hemmen model [51] to describe the interplay of the Kondo effect, spin glass and ferromagnetism in disordered Ce alloys. This model gives a good description of this inter- play and appears to be simpler than the previous models, in particular for the treatment of clusters, as we will see later on [52].

Thus, in order to improve again the agreement with the experimental case of $\mathrm{CeNi}_{1-x} \mathrm{Cu}_{x}$ alloys, we introduce here a new kind of disordered coupling $J_{i j}$ given by van Hemmen (vH) [51] as

$$
J_{i j}=\frac{J}{N}\left(\xi_{i} \eta_{j}+\eta_{i} \xi_{j}\right)+\frac{I_{0}}{N}
$$

where $\xi_{i}$ and $\eta_{j}$ are equal to \pm 1 and are random variables which follow a bimodal distribution [52].

In Eq. (6), we take both a random contribution and a ferromagnetic one, respectively proportional to the constant $J$ and $I_{0}$, which can describe both the SG and the ferromagnetic phases.

We have then studied in detail the different phases obtained by using the $\mathrm{vH}$ approach and we have obtained different phase diagrams which depend on the ratio $I_{0} / J$. Several cases are described in Ref. [52] and Fig. 1 shows here the typical case of a transition with decreasing temperature from spin glass (SG) to ferromagnetism (FE) with an intermediate "mixed" phase $\mathrm{SG}+\mathrm{FE}$ for small $J_{\mathrm{K}}$ values and still the Kondo phase for large $J_{\mathrm{K}}$ values [52]. The use of the van Hemmen model offers some advantages: first, the calculation does not need the use of the replica method and is simpler; second, there exists a real mixed phase between spin glass and ferromagnetism, which is in good agreement with the experimental phase diagram of $\mathrm{CeNi}_{x} \mathrm{Cu}_{1-x}$ alloys [52]. In conclusion of this long study, we can think that the more "local" description [52] given by Eq. (6) seems to be more adequate here than the "average" description used for example in the Sherrington-Kirkpatrick approach [45, 47].

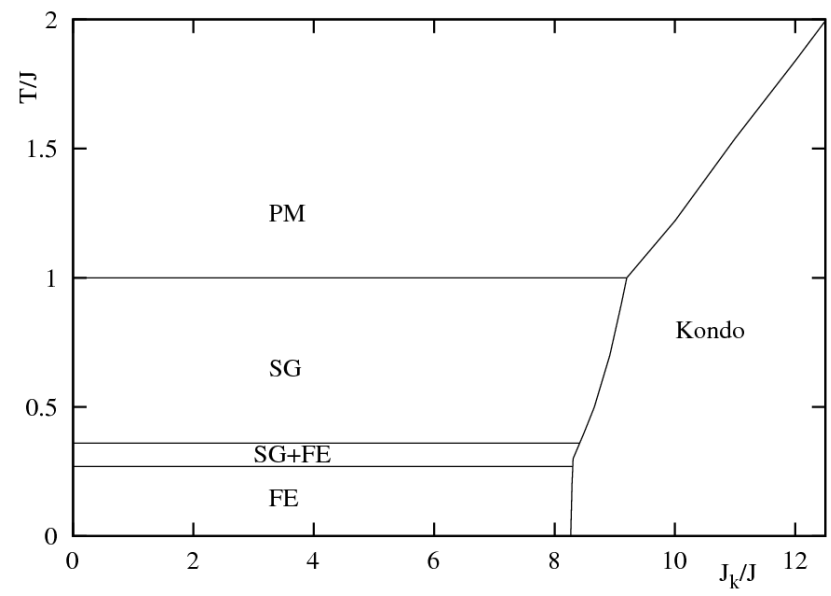

Fig. 1. Phase diagram $T / J$ versus $J_{\mathrm{K}} / J$ for $I_{0} / J=1.4$ in the van Hemmen model according to Ref. [52].

However, a real improvement will be provided by trying to describe better the clusters which are yielding the $\mathrm{CG}$ state with ferromagnetic correlations and the IFM state and then the observed percolative CGIFM transition observed with decreasing temperature 
$[37,38]$. A first approach had been proposed to obtain clusters with intrasite Kondo interactions and both intra- and inter-cluster magnetic interactions within the Sherrington-Kirkpatrick approach [53].

More recently, a Kondo cluster-glass model for spin glass cerium alloys, like $\mathrm{CeNi}_{x} \mathrm{Cu}_{1-x}$ or $\mathrm{CeRh}_{x} \mathrm{Pd}_{1-x}$, has been proposed using the vH model [54]. This model starts from the Kondo and ferromagnetic intra-cluster interactions and an inter-cluster $\mathrm{vH}$ interaction given by Eq. (6). The intra-cluster interactions are treated exactly by diagonalisation and the inter-cluster interaction is treated by the mean field approximation, as previously used for the $\mathrm{vH}$ model. The number of atoms $n_{\mathrm{s}}$ inside each cluster is presently limited to 4 or 5 [54]. However, for relatively large values of $J_{\mathrm{K}}$ and $I_{0}$, we have obtained successively with decreasing temperature a Kondo phase followed by a Kondo cluster-spin glass where both the Kondo and the cluster spin glass (CSG) order parameters are non-zero; finally a mixed KondoCSG-ferromagnetic phase can be obtained at very low temperatures. Thus, the transition with decreasing temperature is rather smooth and can be interpreted as a percolative one, which can present an account for the experimental percolative CG-IFM transition observed in disordered cerium alloys [39, 54].

\section{Conclusions}

Thus, we have presented here several Kondo lattice models which can explain different interplays between the Kondo effect and magnetism, i.e. firstly the Kondo-magnetic order competition and the connected pressure effect observed in many cerium compounds, then the coexistence between ferromagnetism and the underscreened Kondo lattice effect observed in some uranium and neptunium compounds and finally the competition between Kondo, spin glass and magnetic order effects observed in disordered cerium or uranium alloys.

The superconductivity observed in cerium and uranium compounds, like for example $\mathrm{CeCu}_{2} \mathrm{Si}_{2}$ [7, 55] or $\mathrm{UPd}_{2} \mathrm{Al}_{3}$ [56], but also in americium metal [57] and $\mathrm{PuCoGa}_{5}$ [58] compounds, is also a very fascinating subject which is extensively studied at present, as shown in Refs. [5-7] and references therein.

In conclusion, there is still a lot of works in the field of the interplay between the Kondo effect and magnetism, particularly the different properties of actinide systems which can present a multichannel Kondo effect, the superconductivity of these strongly correlated electron systems and the interaction between the spin-glass and Kondo states in disordered cerium or uranium alloys. The recent work of tuning the dimensionality of a heavy fermion compound by reducing the thickness of the layers [19] is very interesting. Indeed, the study of these extremely thin layers or more generally of nanoscale Kondo systems is also extremely promising for the study of the heavy fermion physics.

\section{Acknowledgments}

B. Coqblin acknowledges J.R. Iglesias, C. Lacroix, S.G. Magalhaes, N.B. Perkins, M. Reiffers, A.S. da Rosa Simoes, C. Thomas, and F.M. Zimmer for very fruitful discussions.

\section{References}

[1] S. Doniach, in: Proc. Valence Instabilities and Related Narrow-Band Phenomena, Ed. R.D. Parks, Plenum Press, New York 1976, p. 168.

[2] A.C. Hewson, The Kondo problem to Heavy Fermions, Cambridge University Press, 1993.

[3] J.M. Lawrence, P.S. Risenborough, R.D. Parks, Rep. Prog. Phys. 44, 1 (1981).

[4] G.R. Stewart, Rev. Mod. Phys. 56, 755 (1984).

[5] B. Coqblin, AIP Conf. Proc. 846, 3 (2006).

[6] J. Flouquet, "On the Heavy Fermion Road", arXiv:cond-mat/0501602v1 [cond-mat.str-el].

[7] F. Steglich, Physica B 359-361, 326 (2005).

[8] B. Barbara, H. Bartholin, D. Florence, M.F. Rossignol, E. Walker, Physica B 86-88, 177 (1977).

[9] T. Graf, J.D. Thompson, M.F. Hundley, R. Movshovich, Z. Fisk, D. Mandrus, R.A. Fischer, N.E. Phillips, Phys. Rev. Lett. 78, 3769 (1997).

[10] K. Alami-Yadri, H. Wilhelm, D. Jaccard, Physica B 259-261, 157 (1999).

[11] C. Lacroix, M. Cyrot, Phys. Rev. B 20, 1969 (1979).

[12] P. Coleman, N. Andrei, J. Phys., Condens. Matter 1, 4057 (1989).

[13] J.R. Iglesias, C. Lacroix, B. Coqblin, Phys. Rev. B 56, 11820 (1997).

[14] B. Coqblin, C. Lacroix, M.A. Gusmão, J.R. Iglesias, Phys. Rev. B 67, 064417 (2003).

[15] B. Coqblin, M.A. Gusmão, J.R. Iglesias, A.R. Ruppenthal, C. Lacroix, J. Magn. Magn. Mater. 226-230, 115 (2001).

[16] P. Nozières, Eur. Phys. J. B 6, 447 (1998).

[17] J. Rossat-Mignod, L.P. Regnault, J.L. Jacoud, C. Vettier, P. Lejay, J. Flouquet, E. Walker, D. Jaccard, A. Amato, J. Magn. Magn. Mater. 76-77, 376 (1988).

[18] B. Coqblin, Acta Phys. Pol. A 115, 13 (2009).

[19] H. Shishido, T. Shibauchi, K. Yasu, T. Kato, H. Kontani, T. Terashima, Y. Matsuda, Science 327, 980 (2010).

[20] R. Jullien, M.T. Beal-Monod, B. Coqblin, Phys. Rev. B 9, 1441 (1974).

[21] B. Coqblin, J.R. Iglesias-Sicardi, R. Jullien, Contemp. Phys. 19, 327 (1978).

[22] Q.G. Sheng, B.R. Cooper, J. Mag. Mag. Mater. 164, 335 (1996).

[23] J. Schoenes, O. Vogt, J. Lohle, F. Hulliger, K. Mattenberger, Phys. Rev. B 53, 14987 (1996).

[24] G. Zwicknagl, A.N. Yaresko, P. Fulde, Phys. Rev. B 68, 052508 (2003) 
[25] Z. Bukowski, R. Troc, J. Stepien-Damm, C. Sulkowski, V.H. Tran, J. Alloys Comp. 403, 65 (2005).

[26] V.H. Tran, R. Troc, Z. Bukowski, D. Badurski, C. Sulkowski, Phys. Rev. B 71, 094428 (2005).

[27] E. Colineau, F. Wastin, J.P. Sanchez, J. Rebizant, J. Phys., Condens. Matter 20, 075207 (2008).

[28] V.H. Tran, J.C. Griveau, R. Eloirdi, W. Miiller, E. Colineau, Phys. Rev. B 82, 094407 (2010).

[29] N.B. Perkins, M.D. Nunez-Regueiro, J.R. Iglesias, B. Coqblin, Phys. Rev. B 76, 125101 (2007).

[30] C. Thomas, Acirete S. da R. Simoes, C. Lacroix, J.R. Iglesias, B. Coqblin, Physica B 404, 3008 (2009).

[31] C. Thomas, Acirete S. da R. Simoes, J.R. Iglesias, C. Lacroix, N.B. Perkins, B. Coqblin, to be published.

[32] A.L. Cornelius, J.S. Schilling, O. Vogt, K. Mattenberger, U. Benedict, J. Magn. Magn. Mater. 161, 169 (1996).

[33] P. Link, U. Benedict, J. Wittig, H. Wuhl, J. Phys., Condens. Matter 4, 5585 (1992).

[34] P. Nozières, A. Blandin, J. Phys. (France) 41, 193 (1980).

[35] J.C. Gomez Sal, J. Garcia Soldevilla, J.A. Blanco, J.I. Espeso, J. Rodriguez Fernandez, F. Luis, F. Bartolomé, J. Bartolomé, Phys. Rev. B 56, 11741 (1997).

[36] J. Garcia Soldevilla, J.C. Gomez Sal, J.A. Blanco, J.I. Espeso, J. Rodriguez Fernandez, Phys. Rev. B 61, 6821 (2000).

[37] N. Marcano, J.I. Espeso, J.C. Gomez Sal, J. Rodriguez Fernandez, J. Herrero-Albillos, F. Bartolome, Phys. Rev. B 71, 134401 (2005).

[38] N. Marcano, J.C. Gomez Sal, J.I. Espeso, J.M. De Teresa, P.A. Algarabel, C. Paulsen, J.R. Iglesias, Phys. Rev. Lett. 98, 166406 (2007).

[39] N. Marcano, S.G. Magalhaes, B. Coqblin, J.C. Gómez Sal, J.I. Espeso, F.M. Zimmer, J.R. Iglesias, J. Phys., Conf. Series 200, 012111 (2010).

[40] T. Westerkamp, M. Deppe, R. Kucler, M. Brando, C. Geibel, P. Gegenwart, A.P. Pikul, F. Steglich, Phys. Rev. Lett. 102, 206404 (2009).

[41] M. Brando, T. Westerkamp, M. Deppe, P. Gegenwart, C. Geibel, F. Steglich, J. Phys., Conf. Series 200 012016 (2010).
[42] S. Majumdar, F.V. Sampathkumaran, St. Berger, M. Della Mea, H. Michor, E. Bauer, M. Brando, J. Hemberger, A. Loidl, Solid State Commun. 121, 665 (2002).

[43] R. Vollmer, T. Pietrus, H. v. Lohneysen, R. Chau, M.B. Maple, Phys. Rev. B 61, 1218 (2000).

[44] V.S. Zapf, R.P. Dickey, E.J. Freeman, C. Sirvent, M.B. Maple, Phys. Rev. B 65, 024437 (2002).

[45] D. Sherrington, S. Kirkpatrick, Phys. Rev. Lett. 35, 1792 (1975)

[46] A. Theumann, B. Coqblin, S.G. Magalhaes, A.A. Schmidt, Phys. Rev. B 63, 054409 (2001).

[47] S.G. Magalhaes, A.A. Schmidt, Alba Theumann, B. Coqblin, Eur. Phys. J. B 30, 419 (2002).

[48] S.G. Magalhaes, A.A. Schmidt, F.M. Zimmer, Alba Theumann, B. Coqblin, Eur. Phys. J. B 34, 447 (2003).

[49] D.J. Mattis, Phys. Lett. A 56, 421 (1977).

[50] S.G. Magalhaes, F.M. Zimmer, P.R. Krebs, B. Coqblin, Phys. Rev. B 74, 014427 (2006).

[51] J.L. van Hemmen, J. Phys. C, Solid State Phys. 19, L-379 (1986)

[52] S.G. Magalhaes, F.M. Zimmer, B. Coqblin, Phys. Rev. B 81, 094424 (2010).

[53] F.M. Zimmer, S.G. Magalhaes, B. Coqblin, Physica B 404, 2972 (2009).

[54] F.M. Zimmer, S.G. Magalhaes, B. Coqblin, presented at SCES2010.

[55] F. Steglich, J. Aarts, C.D. Bredl, W. Lieke, D. Meschede, W. Franz, H. Schafer, Phys. Rev. Lett. 43, 1892 (1979).

[56] C. Geibel, C. Schank, S. Thies, H. Kitazawa, C.D. Bredl, A. Bohm, M. Rau, A. Grauel, R. Caspary, R. Helfrich, U. Ahlheim, G. Weber, F. Steglich, Z. Phys. B 84, 1 (1991).

[57] J.L. Smith, R.G. Haire, Science 200, 535 (2005).

[58] J.L. Sarrao, L.A. Morales, J.D. Thomson, B.L. Scott, G.R. Stewart, F. Wastin, J. Rebizant, P. Boulet, E. Colineau, G.H. Lander, Nature 420, 297 (2002). 